\title{
Manejo de las infecciones por gérmenes gram negativos multirresistentes en el Hospital Regional de Villarrica de ene- ro a junio $2019^{3}$ \\ Management of multi-resistant gram-negative germ infec- tions in the Regional Hospital of Villarrica from January to June 2019
}

\begin{abstract}
Mahia Jazmín Irala Espínola ;Mirna Cuevas5; Clara María Irala Benítez; Pablo Alexis Chávez Santacruz; María José Ugarte Rodas; Fiorella Aline Urrustarazu Borja; Cristofer Dalton

Thomas Zoehler; Soraya Benítez Vera ${ }^{6}$.
\end{abstract}

DOI: https://doi.org/10.36003/Rev.investig.cient.tecnol.V3N2(2019)2

\section{RESUMEN}

En el departamento del Guairá, cada día se registran nuevos casos de resistencia a antibióticos la mayoría presentes en bacterias gram negativas multirresistentes en las diversas infecciones que pueden llegar a causar dependiendo de la vía de entrada y factores patogénicos del germen y estado de inmunidad del huésped. Con el objetivo de determinar el manejo terapéutico seguido en el hospital regional de Villarrica frente a las infecciones a gérmenes gram negativos multirresistentes, se realizó un estudio cuantitativo, del tipo descriptivo observacional y corte transversal. Los participantes fueron seleccionados a partir de los datos asentados en el libro de registro de interconsultas del área Infectología Clínica, teniendo un total de 54 casos en el periodo de enero a junio 2019. Los resultados revelaron la existencia de una mayor predisposición en mujeres mayores a 60 años, pacientes diabéticos y quienes eran derivados desde otros servicios, ante las infecciones a gérmenes gram negativos Multirresistentes. La terapéutica resultó ser diferente en cada caso variando según el diagnóstico y el germen aislado, pero se observó una preferencia por la combinación sinérgica de antibióticos de amplio espectro en la mayoría de los casos, además de una duración establecida de entre 14 a 21 días para el manejo de casi todas las infecciones.

Palabras clave: Gérmenes; Antibióticos; Terapéutica.

${ }^{3}$ Fecha de recepción: octubre 2019; fecha de aceptación: noviembre 2019

${ }^{4}$ Estudiante de Medicina de la Facultad de Ciencias de la Salud Universidad Católica, Guairá, Villarrica, Paraguay.

${ }^{5}$ Docente de la Facultad de Ciencias de la Salud Universidad Católica, Guairá, Villarrica, Paraguay.

${ }^{6}$ Estudiantes de Medicina de la Facultad de Ciencias de la Salud Universidad Católica, Guairá, Villarrica, Paraguay. 


\section{ABSTRACT}

In the department of Guairá, new cases of antibiotic resistance are registered every day, most of which are present in multidrug-resistant gram-negative bacteria in the various infections that they can cause depending on the route of entry and pathogenic factors of the germ and state of immunity of the host. In order to determine the therapeutic management followed in the regional hospital of Villarrica against infections to multiresistant gram-negative germs, a quantitative study was carried out, of the observational descriptive type and cross section. The participants were selected from the data established in the interconsultation record book of the Clinical Infectology area, having a total of 54 cases in the period from January to June 2019. The results revealed the existence of a greater predisposition in women older than 60 years, diabetic patients and those who were referred from other services, before infections to gram-negative multiresistant ger$\mathrm{ms}$. The therapy turned out to be different in each case varying according to the diagnosis and the isolated germ, but a preference was observed for the synergistic combination of broad-spectrum antibiotics in most cases, in addition to an established duration of between 14 to 21 days for the management of almost all infections.

Keywords: Germs; Antibiotics; Therapy. 


\section{INTRODUCCIÓN}

La elevada incidencia de bacterias mutiresistentes y su asociación con padecimientos de alta morbilidad1 a causa de fallas terapéuticas, la convierten en un problema de salud pública a nivel nacional, que requiere mucha atención, al mismo tiempo existen varios factores que deben tenerse en cuenta.

Las enfermedades son de difícil remisión justamente por el agente etiológico implicado y su respuesta natural intrínseca o adquirida frente a los antibióticos. La producción de beta-lactamasas de espectro extendido es el mayor problema actúa (1, 2) de resistencia entre las enterobacterias, que causan infecciones nosocomiales, pero que también se están aislando en pacientes no ingresados (3).

En la última década se generó un aumento de la incidencia de infecciones causadas por bacterias gramnegativas resistentes a múltiples fármacos, incluyendo enterobacterias multirresistentes (MR), Pseudomonas aeruginosa-MR y Acinetobacter baumannii-MR $(4,5)$.

El identificar los parámetros correctos de la terapéutica que posean resultados eficaces de erradicar la infección presente, resulta un problema desafiante pues los gérmenes implicados demuestran alta resistencia con la posterior falla de tratamiento o constantes recidivas.

Este aumento de resistencias antimicrobianas, unido al poco desarrollo de nuevos antibióticos, hace que cada vez se disponga de menos opciones terapéuticas para el tratamiento de dichas enfermedades infecciosas (6).

Siendo la principal problemática determinar el manejo terapéutico de infecciones por gérmenes gram negativos multirresistentes, utilizado en el Hospital Regional Villarrica, fue tomada la iniciativa para realizar la presente investigación.
Las bacterias gram negativas multirresistentes son un grave problema de salud en todo el mundo (7). Ello se relaciona con la gravedad de las infecciones que pueden causar, las dificultades para establecer un tratamiento empírico, e incluso dirigido, correcto, la facilidad para la dispersión de la multirresistencia y la ausencia de nuevos antimicrobianos activos frente a estos patógenos.

Las enfermedades causadas por estos gérmenes se encuentran afectando indistintamente a hombres, mujeres y niños. Estos en su gran mayoría se encuentran en condiciones de vida donde constantemente viven expuestos a un ambiente hospitalario por motivos varios (8), con la posterior colonización y/o infección por estas bacterias y con una inmunidad comprometida y/o insuficiente. Cabe destacar que la carencia de medidas higiénicas incluso en los centros de salud y el contexto socioeconómico y educacional precarios de los pacientes, son condicionantes que dificultan en medida notable el éxito de la terapéutica establecida.

En el departamento del Guairá, lo anteriormente expuesto constituye un problema de Salud Pública, ya que no se encuentra exento de las notificaciones de casos de infecciones a bacterias multirresistentes en donde la terapéutica resulta no ser la más adecuada.

Impulsado por lo ya expuesto, la presente investigación tuvo como objetivo determinar el manejo terapéutico utilizado en el Hospital Regional de Villarrica del Espíritu Santo frente a las infecciones a gérmenes gram negativos multirresistentes que tuvieron lugar en el área de clínica médica durante los meses de enero a junio del 2019. Como objetivos específicos se plantearon los siguientes: caracterizar a la población según edad y sexo; identificar comorbilidades de pacientes en estudio; describir antecedentes de internación 
en otros servicios en el Hospital Regional de Villarrica; clasificar según diagnóstico; describir el tratamiento implementado se-

\section{MÉTODO}

Se basó en un estudio cuantitativo del tipo observacional descriptivo y corte transversal, de modo a determinar el manejo terapéutico de las infecciones a gérmenes gram negativos multirresistentes en el Hospital Regional de Villarrica de enero a junio del 2019. El estudio ha sido retrospectivo, puesto que los datos fueron recolectados del libro de interconsultas del departamento de infectología clínica del Hospital Regional de Villarrica del Espíritu, durante los meses de enero a junio del 2019.

Participantes: los pacientes con infección a gérmenes gram negativos multirresistentes registrados en el libro de interconsultas del departamento de infectología clínica del Hospital Regional de Villarrica del Espíritu, durante los meses de Enero a Junio del 2019. Estos totalizaron un número 54 pacientes.

Para el abordaje del estudio de investi- gún aislamiento y sensibilidad del germen; y definir la duración del tratamiento según el diagnóstico establecido.

gación se procedió elaborar una matriz de recolección de información donde fueron traspasados los datos a partir del libro de interconsultas del departamento de infectología clínica del Hospital Regional de Villarrica del Espíritu

Para el procesamiento estadístico se diseñó una base de datos por el sistema EXCEL. Toda la información recabada fue procesada y analizada, usando herramientas estadísticas descriptivas. Se utilizaron medidas de tendencia central, porcentaje (\%) y los gráficos de tablas y proporciones.

\section{Consideraciones éticas.}

Se procedió a la solicitud por escrito a las autoridades locales de turno para acceder a los registros asentados dentro del libro de interconsultas del departamento de infectología clínica del Hospital Regional de Villarrica del Espíritu. La identidad de los pacientes fueron resguardados bajo absoluta confidencialidad.

\section{RESULTADOS}

En el primer rango etario según edades, que abarca desde 1 a 29 años, se observan 4 pacientes correspondiendo al 7,4\%. En el segundo rango, que va desde 30 a 59 años, se haya 22 pacientes, equivalentes al 40,7\%. Y en el tercer rango, abarcando desde 60 a 90 años, se encuentran 28 pacientes correspondiendo al 51,8\%. El promedio de edad es de 63 años.

Gráfico 1. Caracterización de los pacientes con infecciones a gérmenes gram negativos multirresistentes según edad.

Fuente: Datos asentados en el libro de registro de interconsultas del área Infectología Clínica.
1-29 años

30-59 años

60-90 años




Se presenta un total de 54 pacientes, del cual 32 son mujeres correspondiendo al 59,2\% de la totalidad, y 22 son hombres equivaliendo al $40,7 \%$ restante.

Gráfico 2. Caracterización de los pacientes con infecciones a gérmenes gram negativos multirresistentes según sexo.

Fuente: Datos asentados en el libro de registro de interconsultas del área Infectología Clínica


Del total de 54 pacientes, solo 20 presentan comorbilidades por lo que corresponden al 37,0\%, y el $62,9 \%$ restante representa a los pacientes sin comorbilidades.

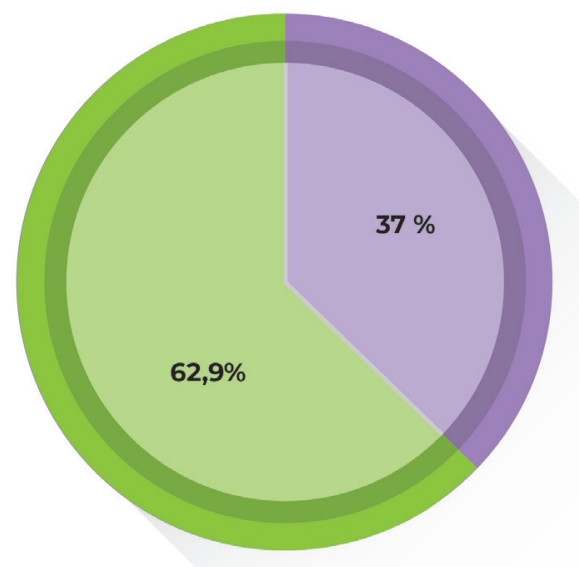

Sin comorbilidades

Comorbilidades

Gráfico 3. Presencia de comorbilidades en los pacientes con infecciones a gérmenes gram negativos multirresistentes.

Fuente: Datos asentados en el libro de registro de interconsultas del área Infectología Clínica

De los 20 pacientes con comorbilidades se observa que la más prevalente es la Diabetes y sus complicaciones, coincidente en 6 de los casos. El segundo lugar lo ocupan el Traumatismo cráneo-encefálico y Accidente Cerebro Vascular ambos con 4 casos. El último lugar es para la Carcinomatosis Peritoneal, la Leucemia y la Enfermedad Pulmonar Obstructiva Crónica, encontrados cada uno solo en 2 casos.

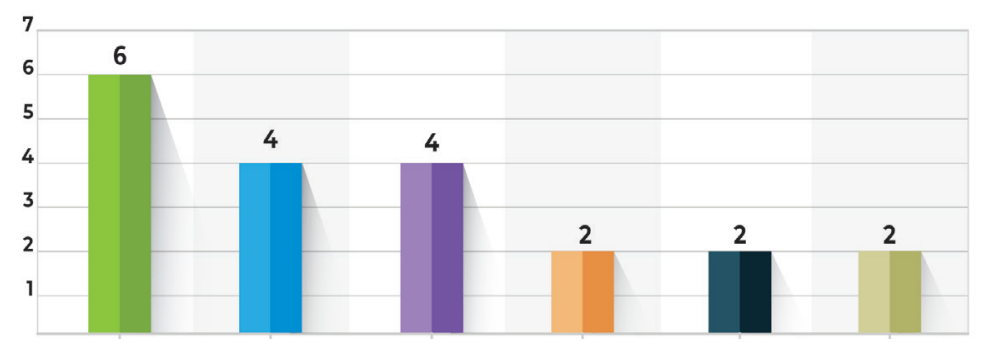

Gráfico 4. Clasificación de las comorbilidades halladas en pacientes con infecciones a gérmenes gram negativos multirresistentes.

Fuente: Datos asentados en el libro de registro de interconsultas del área Infectología Clínica. 
De los 20 pacientes con comorbilidades se observa que la más prevalente es la Diabetes y sus complicaciones, coincidente en 6 de los casos. El segundo lugar lo ocupan el Traumatismo cráneo-encefálico y Accidente Cerebro Vascular ambos con 4 casos. El último lugar es para la Carcinomatosis Peritoneal, la Leucemia y la Enfermedad Pulmonar Obstructiva Crónica, encontrados cada uno solo en 2 casos.

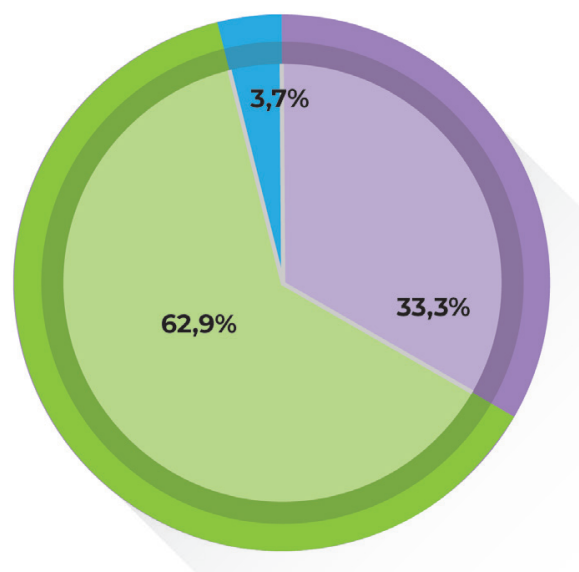

Sin comorbilidades

Comorbilidades

Gráfico 3. Presencia de comorbilidades en los pacientes con infecciones a gérmenes gram negativos multirresistentes.

Fuente: Datos asentados en el libro de registro de interconsultas del área Infectología Clínica

El diagnóstico prevalente es la Infección de vías urinarias (IVU) a Klebsiella pneumoniae multirresistente (MR), encontrada en 12 casos correspondiendo al $22,2 \%$ del total. Sin embargo, haciendo caso omiso al diagnóstico patológico, se observa que la Pseudomona aeruginosa MR es el germen aislado de la mayoría de las infecciones con un $36,9 \%$ sobre el total.

Tabla 1. Distribución según el diagnóstico y germen aislado

\begin{tabular}{lcc}
\hline Diagnóstico & Cantidad de Pacientes & Porcentaje \\
\hline $\begin{array}{l}\text { Sepsis a punto de partida } \\
\text { pulmonar a Pseudomonas } \\
\text { aeruginosa MR }\end{array}$ & 6 & $11,1 \%$ \\
$\begin{array}{l}\text { IVU a Klebsiella } \\
\text { pneumoniae MR }\end{array}$ & 12 & $22,2 \%$ \\
$\begin{array}{l}\text { IVU a Klebsiella } \\
\text { pneumoniae MR }\end{array}$ & 8 & $14,8 \%$ \\
$\begin{array}{l}\text { Neumonía a Pseudomonas } \\
\text { aeruginosa MR }\end{array}$ & 10 & $18,5 \%$ \\
$\begin{array}{l}\text { Bacteriemia a Pseudomonas } \\
\text { aeruginosa }\end{array}$ & 2 & $3,7 \%$ \\
$\begin{array}{l}\text { Infección de piel y partes } \\
\text { blandas a Pseudomonas } \\
\text { aeruginosa }\end{array}$ & 2 & $3,7 \%$ \\
$\begin{array}{l}\text { Neumonía a Klebsiella } \\
\text { pneumoniae }\end{array}$ & 4 & $7,4 \%$
\end{tabular}


Sepsis a punto de partida urinario a Eschericcia

coli BLEE

Neumonía asociada a

ventilación a Acinetobacter

baumannii

Shock séptico a punto de partida urinario Klebsiella pneumoniae MR

\begin{tabular}{lcc}
\hline Total & 54 & $100 \%$ \\
\hline
\end{tabular}

Fuente: Datos asentados en el libro de registro de interconsultas del área Infectología Clínica.

Se observa que el fármaco destinado para el tratamiento varía no solo de acuerdo al agente causal sino también al diagnóstico establecido. Se aprecia el predominio de la terapia combinada que fue la elegida en el $62,9 \%$ de los casos, siendo preferida en el $37,1 \%$ restante la monoterapia con Meropemen o Imipenem.

Tabla 2. Clasificación de la terapéutica seleccionada según diagnóstico y germennaislado.

\begin{tabular}{|c|c|c|c|c|}
\hline \multirow{2}{*}{ Diagnóstico } & \multicolumn{4}{|c|}{ Aislamiento } \\
\hline & $\begin{array}{l}\text { Klebsiella } \\
\text { pneumoniae } \\
\text { multirresistente }\end{array}$ & $\begin{array}{l}\text { Pseudomonas } \\
\text { aeruginosa }\end{array}$ & $\begin{array}{l}\text { Escherichia } \\
\text { coli blee }\end{array}$ & $\begin{array}{l}\text { Acinetobacter } \\
\text { baumannii }\end{array}$ \\
\hline $\begin{array}{l}\text { Sepsis a punto de } \\
\text { partida pulmonar }\end{array}$ & Meropenem & $\begin{array}{l}\text { Piperacilina- } \\
\text { Tazobactam }+ \\
\text { Amikacina }\end{array}$ & & \\
\hline $\begin{array}{l}\text { Infección de vías } \\
\text { urinarias }\end{array}$ & Meropenem & & $\begin{array}{l}\text { 1. Imipenem } \\
\text { 2. Piperacilina - } \\
\text { Tazobactam }\end{array}$ & \\
\hline Neumonía & Meropenem & $\begin{array}{l}\text { 1. } \text { Meropenem }+ \\
\text { Colistina } \\
\text { 2. Piperacilina }+ \\
\text { tazobactam } \\
\end{array}$ & & \\
\hline $\begin{array}{l}\text { Sepsis a punto de } \\
\text { partida urinario }\end{array}$ & & & Imipenem & \\
\hline $\begin{array}{l}\text { Neumonía } \\
\text { asociada a } \\
\text { ventilación }\end{array}$ & Imipenem & $\begin{array}{l}\text { Meropenem + } \\
\text { Colistina }\end{array}$ & & $\begin{array}{l}\text { Meropenem + } \\
\text { Colistina }\end{array}$ \\
\hline Bacteriemia & & $\begin{array}{l}\text { Piperacilina - } \\
\text { Tazobactam }\end{array}$ & & \\
\hline $\begin{array}{l}\text { Infección de piel } \\
\text { y partes blandas }\end{array}$ & & Meropenem & & \\
\hline $\begin{array}{l}\text { Shock séptico a } \\
\text { punto de partida } \\
\text { urinario }\end{array}$ & $\begin{array}{l}\text { Meropenem + } \\
\text { Colistina }\end{array}$ & & & \\
\hline
\end{tabular}

Fuente: Datos asentados en el libro de registro de interconsultas del área Infectología Clínica. 
Respecto a la duración del tratamiento, existe una variación relacionada al germen asilado y al diagnóstico establecido para cada paciente, sin embargo, existe una duración prevalente de 14 a 21 días observada en el 81,4\% de los casos.

Tabla 3. Duración del tratamiento según diagnóstico y germen aislado.

\begin{tabular}{|c|c|c|c|c|c|}
\hline \multirow{2}{*}{ Diagnóstico } & \multicolumn{4}{|c|}{ Aislamiento } & \multirow{2}{*}{$\begin{array}{c}\text { Días de } \\
\text { Tratamientos }\end{array}$} \\
\hline & $\begin{array}{c}\text { Klebsiella } \\
\text { pneumoniae } \\
\text { multirresistente }\end{array}$ & $\begin{array}{l}\text { Pseudomonas } \\
\text { aeruginosa }\end{array}$ & $\begin{array}{l}\text { Escherichia } \\
\text { coli blee }\end{array}$ & $\begin{array}{c}\text { Acinetobacter } \\
\text { baumannii }\end{array}$ & \\
\hline $\begin{array}{l}\text { Sepsis a punto de } \\
\text { partida pulmonar }\end{array}$ & 1. Meropenem & $\begin{array}{l}\text { 2. Piperacilina - } \\
\text { Tazobactam }+ \\
\text { Amikacina }\end{array}$ & & & $\begin{array}{l}\text { 1. } 14 \text { días } \\
\text { 2. } 21 \text { días }\end{array}$ \\
\hline $\begin{array}{l}\text { Infección de vías } \\
\text { urinarias }\end{array}$ & 1. Meropenem & & $\begin{array}{l}\text { 2. Imipenem } \\
\text { 3. Piperacilina } \\
\text { - Tazobactam }\end{array}$ & & $\begin{array}{l}\text { 1. } 14 \text { días } \\
\text { 2. } 14 \text { días } \\
\text { 3. } 10 \text { días }\end{array}$ \\
\hline Neumonía & 1. Meropenem & $\begin{array}{l}\text { 2. } \begin{array}{l}\text { Meropenem }+ \\
\text { Colistina } \\
\text { 3. Piperacilina }+ \\
\text { tazobactam }\end{array} \\
\end{array}$ & & & $\begin{array}{l}\text { 1. } 07 \text { días } \\
\text { 2. } 21 \text { días } \\
\text { 3. } 21 \text { días }\end{array}$ \\
\hline $\begin{array}{l}\text { Sepsis a punto de } \\
\text { partida urinario }\end{array}$ & & & Imipenem & & 14 días \\
\hline $\begin{array}{l}\text { Neumonía } \\
\text { asociada a } \\
\text { ventilación }\end{array}$ & 1. Imipenem & $\begin{array}{l}\text { 2. Meropenem } \\
+ \text { Colistina }\end{array}$ & & $\begin{array}{l}\text { Meropenem } \\
+ \text { Colistina }\end{array}$ & $\begin{array}{l}\text { 1. } 14 \text { días } \\
\text { 2. } 21 \text { días } \\
\text { 3. } 14 \text { días }\end{array}$ \\
\hline Bacteriemia & & $\begin{array}{l}\text { Piperacilina - } \\
\text { Tazobactam }\end{array}$ & & & 21 días \\
\hline $\begin{array}{l}\text { Infección de piel } \\
\text { y partes blandas }\end{array}$ & & Meropenem & & & 10 días \\
\hline $\begin{array}{l}\text { Shock séptico a } \\
\text { punto de partida } \\
\text { urinario }\end{array}$ & $\begin{array}{l}\text { Meropenem + } \\
\text { Colistina }\end{array}$ & & & & 21 días \\
\hline
\end{tabular}

Fuente: Datos asentados en el libro de registro de interconsultas del área Infectología Clínica.

\section{DISCUSIÓN}

De acuerdo a los resultados, y en concordancia con otros estudios (9), se obtuvo que, al caracterizar a la población según edad y sexo, el sexo femenino y aquellos con una edad mayor a 60 años fueron los que con mayor frecuencia presentaron infecciones a gérmenes gram negativos multirresistentes (MR).

Es llamativa la carencia de alguna enfermedad de base registrada, en la mayoría de los pacientes participantes del estudio, que pudiera representar un factor predisponente frente a las infecciones, en consecuencia, debe indagarse acerca de otros factores diferentes a los abarcados en el estudio, como la frecuencia de internación al año de los pacientes u otras costumbres relevantes que aumenten la predisposición de los mismos.

Entre quienes presentaban alguna co- 
morbilidad, en orden de frecuencia, la diabetes ocupaba el primer lugar, seguido de accidente cerebro vascular, traumatismo craneoencefálico, carcinomatosis peritoneal, leucemia y enfermedad pulmonar obstructiva crónica.

Respecto a los antecedentes de internación, se constató que los pacientes provenientes de la Unidad de Terapia Intensiva y con diagnóstico de infección de la vía urinaria (IVU) hacían, con más frecuencia, infecciones a bacterias gram negativas MR que los derivados desde otros servicios y con diagnósticos diferentes.

La Pseudomona aeruginosa MR fue la bacteria aislada de la mayoría de las infecciones, lo cual concuerda con estudios que revelan una tendencia mayor de aparición de resistencia en bacterias gram negativas. Además, también se aislaron en los pacientes Klebsiella pneumoniae MR, Escherichia coli blee, Acinetobacter baumannii (10).

En cuanto al manejo terapéutico para estos pacientes, se apreció la utilización de antibióticos de amplio espectro para la todos los casos, y, si bien, el fármaco especifico seleccionado para cada persona variaba en correspondencia a su caso en particular, se resalta una preferencia por la antibioterapia combinada en el manejo de estas infecciones. Esto último es una muestra de una correcta medida para el tratamiento de gérmenes $M R$, pues se ha visto que la monoterapia tiende a resultar menos eficaz $(11,12)$.

La duración del tratamiento también fue distinta dependiendo de la bacteria aislada y del diagnóstico patológico establecido para cada circunstancia, sin embargo, se percibió un predominio en los tratamientos con una duración de 14 días en promedio en los casos de IVU y de 21 días en los casos de neumonía sin hacer distinción en aquellos asociados a ventilación de los demás tipos.

La multirresistencia bacteriana constituye un desafío diagnóstico y terapéutico dado las escasas opciones de antimicrobianos activos frente a las infecciones causadas por estos agentes (13). A esto se le suma la mala utilización de los fármacos efectivos por parte del personal de blanco, que culmina posteriormente con generación de cepas resistentes (14), limitando cada vez más las medidas terapéuticas disponibles para hacerle frente a las infecciones de esta naturaleza.

Un manejo correcto de los algoritmos terapéuticos, basado en el correcto aislamiento del agente infeccioso, la realización del antibiograma (15), y el seguimiento paso a paso del paciente, es la llave que permitiría no solo combatir eficazmente la enfermedad sino también evitar la aparición de gérmenes multirresistentes.

\section{CONCLUSIONES}

Al caracterizar según edad y sexo, se obtuvo que la mayoría de los pacientes eran mujeres mayores de 60 años, con una edad promedio de 63 años, que no registraban en la base de datos comorbilidades.

En la minoría que presentaba alguna enfermedad de base se encontró en orden decreciente de frecuencia diabetes, accidente cerebro vascular, traumatismo craneoencefálico, carcinomatosis peritoneal, leucemia y enfermedad pulmonar obstructiva crónica respectivamente.

Se constató con relación a los antecedentes de internación que aquellos pacientes provenientes de la Unidad de Terapia Intensiva y diagnosticados con infecciones de la vía urinaria, hacían infecciones a bacterias gram negativas MR con más frecuencia que los derivados desde otros servicios y con diagnósticos diferentes. 
Los gérmenes aislados en orden decreciente de frecuencia fueron la Pseudomona aeruginosa MR, Klebsiella pneumoniae MR, Escherichia coli blee, Acinetobacter baumannii.

Con relación al plan terapéutico, se observó para todos los casos la elección de antibióticos de amplio espectro y de an- tibioterapia combinada como estrategia principal, presentándose variaciones en el fármaco y las combinaciones elegidas dependiendo de las condiciones particulares de cada paciente. En cuanto a la duración del tratamiento se constató que en general oscilaba entre 14-21 días.

\section{BIBLIOGRAFÍA}

1. Martínez, P. Emergencia de bacilos gram negativos multirresistentes: Impacto de las Betalactamasas de espectro extendido y metalo Betalactamasas en hospitales de la Costa Colombiana. Revista MVZ Cordoba [Internet]. 2004 [cited 2019 Oct 6];(2). Available from: http://search.ebscohost.com/login.aspx?direct $=$ true $\& \mathrm{db}=$ edsdoj\&AN=edsdoj.4712679904cb4b70afa53e93b421c79f\&lang=es\&site=eds-live

2. Yábar MN, Curi-Pesantes B, Torres CA, Calderón-Anyosa R, Riveros $\mathrm{M}$, Ochoa TJ. MULTIRRESISTENCIA Y FACTORES ASOCIADOS A LA PRESENCIA DE BETALACTAMASAS DE ESPECTRO EXTENDIDO EN CEPAS DE Escherichia coli PROVENIENTES DE UROCULTIVOS. Revista Peruana de Medicina Experimental y Salud Pública [Internet]. 2017 Oct [cited 2019 Oct 6];34(4):660-5. Available from: http:// search.ebscohost.com/login.aspx?direc$\mathrm{t}=$ true \& $\mathrm{db}=1$ th \& $\mathrm{AN}=134352493 \&$ lan$\mathrm{g}=\mathrm{es} \& \mathrm{site}=\mathrm{eds}-\mathrm{live}$

3. Murray PR, Pfaller MA, Rosenthal KS. Microbiología Médica + StudentConsult [Internet]. Vol. 7.a ed. Barcelona: Elsevier; 2014 [cited 2019 Oct 6]. Available from: http://search.ebscohost. com $/ \log$ in. aspx ?direct $=$ true $\& d b=$ nlebk\&AN=974483\&lang=es\&site=eds-live

4. Briceño, D., Correa, A., Valencia,
C., Torres, J., Pacheco, R., Montealegre, C., et al. Actualización de la resistencia a antimicrobianos de bacilos Gram negativos aislados en hospitales de nivel III de Colombia: años 2006, 2007 y 2008. Biomédica: revista del Instituto Nacional de Salud [Internet]. 2010 [cited 2019 Oct 6];(3):371. Available from: http://search. ebscohost.com/login.aspx?direct=true \&$\mathrm{db}=$ edsdoj\&AN=edsdoj.97f654f05c2d4b89a53a4d1712f942dd\&lang=es\&site $=$ eds-live

5. OMS. Resistencia a los antimicrobianos [Internet]. 2018 Feb [cited 2019 Oct 6]. Available from: https://www.who. int/es/news-room/fact-sheets/detail/resistencia-a-los-antimicrobianos

6. Lombardi, V. ¿Por qué no tenemos nuevos antibióticos? Página oficial de la Universidad Nacional de San Martín [Internet]. 2016 Jul [cited 2019 Oct 6]. Available from: http://www.unsam.edu.ar/tss/ por-que-no-se-descubren-nuevos-antibioticos/ 0

7. Gómez, K. Bacterias multirresistentes: Tratamientos disponibles. Biomedicina [Internet]. 2015 Dec [cited 2019 Oct 6];10(2):6-16. Available from: http:// search.ebscohost.com/login.aspx?direc$\mathrm{t}=$ true \& $\mathrm{db}=$ asn \&AN $=115447968 \&$ authtype $=$ sso\&custid $=$ ns $021105 \&$ lang $=$ es\&site $=$ eds-live 
8. Márquez-Herrera, K., Rojas-Vega, A., Camacho-Moreno, G. Caracterización de pacientes con aislamiento de Klebsiella productora de carbapenemasa en un hospital pediátrico de tercer nivel de Bogotá, Colombia. INFECTIO [Internet]. 2016 Oct 1 [cited 2019 Oct 6];20(4):231-7. Available from: http://search.ebscohost. com/login.aspx ?direct $=$ true $\& d b=$ edsel$\mathrm{p} \& \mathrm{AN}=\mathrm{S} 0123939216000060 \&$ lang $=\mathrm{es} \&$ site $=$ eds-live

9. Jiménez, J., Gaviria, M., Balparda, J., Castrillón, D., Marín, E., Vélez, E., et al. Caracterización clínica, microbiológica y de sensibilidad a antimicrobianos en pacientes con infección nosocomial del tracto urinario: cuatro años y medio de vigilancia epidemiológica. MedUNAB [Internet]. 2011 Dec [cited 2019 Oct 6];14(3):145-50. Available from: http:// search.ebscohost.com/login.aspx?direc$\mathrm{t}=$ true $\& \mathrm{db}=\mathrm{asn} \& \mathrm{AN}=75283542 \&$ lan$\mathrm{g}=\mathrm{es} \&$ site $=\mathrm{eds}-\mathrm{live}$

10. Rincón-León, H.A., Navarro-Fuentes, K.R. Tendencias de resistencia antimicrobiana en patógenos aislados de infecciones nosocomiales. Revista Medica del IMSS [Internet]. 2016 Jan [cited 2019 Oct 6];54(1):32-41. Available from: http:// search.ebscohost.com/login.aspx?direc$\mathrm{t}=$ true $\& \mathrm{db}=$ asn \&AN=112752582\&lan$\mathrm{g}=\mathrm{es} \& \mathrm{site}=\mathrm{eds}-\mathrm{live}$

11. Miranda, J.L., Caballero, A.I., Martínez, P.P., Jiménez, F.P., Lista, J.D. Guía de atención rápida en clínicas médicas [Internet]. Barcelona: Elsevier; 2014 [cited 2019 Oct 6]. Available from: http:// search.ebscohost.com/login.aspx?direct=true $\& \mathrm{db}=$ nlebk\&AN=973265\&lang $=$ es \&site $=$ eds-live

12. Monté Cepero, L., Martínez Casanueva, R. Sensibilidad antimicrobiana de aislamientos en pacientes ingresados en el hospital "Salvador Allende" entre agos- to y diciembre de 2015. Revista Cubana de Higiene y Epidemiología [Internet]. 2017 Apr [cited 2019 Oct 6];55(2):31-45. Available from: http://search.ebscohost. com/login.aspx ?direct $=$ true $\& \mathrm{db}=$ as $n \& A N=137009055 \&$ authtype $=$ sso \& custi$\mathrm{d}=$ ns021105\&lang $=$ es\&site $=$ eds-live

13. Monté Cepero, L., Martínez Casanueva, R. Sensibilidad antimicrobiana8 de aislamientos en pacientes ingresados en el hospital "Salvador Allende" entre agosto y diciembre de 2015. Revista Cubana de Higiene y Epidemiología [Internet]. 2017 Apr [cited 2019 Oct 6];55(2):31-45. Available from: http://search.ebscohost. com/login.aspx?direct $=$ true $\& \mathrm{db}=$ as $\mathrm{n} \& \mathrm{AN}=137009055 \&$ authtype $=$ sso \&custi$\mathrm{d}=$ ns021105\&lang $=$ es\&site $=$ eds-live

14. Flórez Beledo, J., Mediavilla Martínez, A., Armijo Simón, J. Farmacología humana [Internet]. Vol. 6th ed. London: Elsevier Masson; 2013 [cited 2019 Oct 6]. Available from: http://search.ebscohost.com/login.aspx?direct $=$ true $\& \mathrm{db}=$ nlebk\&AN=974488\&lang $=$ es\&site $=$ eds-live

15. Riveros-Pérez, E., Manrique-Abril, F., Ospina Díaz, J. Evaluación de terapia antimicrobiana empírica y adaptación a antibiograma en una clínica de tercer nivel de Tunja. Medicina UPB [Internet]. 2014 Jan [cited 2019 Oct 6];33(1):11-8. Available from: http:// search.ebscohost.com/login.aspx?direc$\mathrm{t}=$ true \& $\mathrm{db}=$ asn \&AN=113999205\&lan$\mathrm{g}=\mathrm{es} \& \mathrm{site}=\mathrm{eds}-\mathrm{live}$ 
1. A.P.A.- Asociación de Psicólogos Americanos (2010). Manual de estilo de publicaciones. (3a.ed.) México: El Manual Moderno.

2. Briones, Y. (2017). Habilidades sociales según el género en estudiantes del nivel secundario del Colegio Adventista Puno, 2017. Universidad Peruana Union, Juliaca. Recuperado el 08 de mayo de 2019, de https://repositorio.upeu.edu.pe/ bitstream/handle/UPEU/702/Yesenia_Tesis_bachiller_2017.pdf?sequence $=1 \&$ is Allowed $=\mathrm{y}$

3. Caballo, V. (2007). Manual de evaluación y entrenamiento de las habilidades sociales. Madrid: Siglo XXI.

4. Eceiza, M., Arrieta, M., \& Goñi, A. (2008). Habilidades Sociales y Contextos de la Conducta Social. Revista de Psicodidáctica, 11-26.

5. Gismero, E. (2010). Escala de habilidades sociales . Madrid: TEA ediciones

6. Hernandez, R., Fernandez, C., \& Baptista, P. (2007). Fundamentos de la metodologia de la investigación. Madrid: McGraw-Hill.

7. Kelly, J. (2002). Entrenamiento en las habilidades sociales (Séptima ed.). Bilbao: Desvlée de Brouwer.

8. Morris, C., \& Maisto, A. (2005). Psicologia (duodécima ed.). Naucalpan de Juárez: Pearson educación de México.

9. Sampieri, H., Callado, C., Baptis- ta, P. (2010). Metodología de la investigación (Quinta Edicion). México D.F.: McGraw-Hill .

9. Zavala, M., Valadez, M., \& Vargas, M. (2008). Revista Electrónica de Investigación Psicoeducativa. Recuperado el 16 de Febrero de 2015, de http:// www.investigacion-psicopedagogica.org/ revista/new/ContadorArticulo.php?271. 